\title{
Timing of optimal surgical intervention for vitreous hemorrhage in patients with proliferative diabetic retinopathy
}

\section{Li Lin}

Wenzhou Medical University Eye Hospital

\section{Yiqi Chen}

Wenzhou Medical University Eye Hospital

\section{Lin Wang}

Wenzhou Medical University Eye Hospital

Lijun Shen ( $\nabla$ eye_slj@163.com )

Wenzhou Medical University Eye Hospital

Research article

Keywords: diabetic retinopathy; vitreous hemorrhage; vitrectomy; timing of surgery

Posted Date: March 11th, 2020

DOI: https://doi.org/10.21203/rs.3.rs-16666/v1

License: (c) (i) This work is licensed under a Creative Commons Attribution 4.0 International License.

Read Full License 


\section{Abstract}

Background $\triangle \mathrm{T}$ o explore the optimal timing of vitrectomy for vitreous hemorrhage in the patients with proliferative diabetic retinopathy (PDR).

Method $₫$ Seventy-six PDR patients (85 eyes) who were diagnosed with vitreous hemorrhage and received vitrectomy were retrospectively enrolled into this study. The patients were categorized into 4 groups based on the timing of surgical intervention after the occurrence of hemorrhage, namely Group 1 (less than 15 days, with 28 eyes of 27 patients), Group 2 (15 30 days, with 29 eyes of 27 patients), Group 3 (30 90 days, with 14 eyes of 11 patients) and Group 4 ( $>90$ days, with 14 eyes of 11 patients). Best corrected visual acuity (BCVA) and central macular thickness (CMT) from OCT before and at 1 mo and 3 mo after operation were collected as the main outcomes. The postoperative complications were also reported.

Result: 1) BCVA (logMAR): the mean pre-operation BCVA was not significantly different among the 4 groups $(P=0.139)$, while the mean post-operation BCVA was significantly worse in groups with longer gap between the vitreous bleeding and the vitrectomy, both at $1 \mathrm{~m}$ and $3 \mathrm{~m}$ after surgery $(P=0.043 ; P<0.001) .2)$ $\mathrm{CMT}(\mu \mathrm{m})$ : Although CMT was unavailable to measure in most included eyes before surgery, the mean CMT was significantly thicker in groups with postponed surgical intervention both at 1-month $(P=0.001)$ and at 3-month ( $\mathrm{P}=0.006)$ after surgery. 3) Complications: No severe complications were spotted in early vitrectomy groups (Group 1 and Group 2) during the follow-up period. At 3 months after the operation, three cases of ocular hypertension and another case of neovascular glaucoma in Group 3 and 4 cases of ocular hypertension and another 3 cases of neovascular glaucoma in Group 4 were observed.

Conclusion: Early vitrectomy is more effective in improving BCVA and decreasing CMT in the PDR patients with vitreous hemorrhage. Moreover, the post-operation complications were spotted less in early vitrectomy groups. Thus we suggested that vitrectomy should be performed early and better within one month after the onset of vitreous hemorrhage in PDR patients.

\section{Background}

The incidence of diabetic retinopathy in diabetic patients is reported to be as high as $38 \%$ with an upward trend ${ }^{[1]}$. Late complications of proliferative diabetic retinopathy (PDR) include vitreous hemorrhage, traction retinal detachment, traction-induced rhegmatogenous retinal detachment, and severe fibrovascular proliferation ${ }^{[2]}$. Vitreous hemorrhage is a severe complication of PDR, which can damage the patient's visual function and lead to consequent problems. Currently, methods to treat vitreous hemorrhage in clinical practice consist of controlling blood sugar and blood pressure, medical treatment and vitrectomy ${ }^{[3]}$.

Vitrectomy is an effective method in treating vitreous hemorrhage. Most studies have confirmed that early vitrectomy can shorten the proliferative lesions, improve the postoperative visual prognosis and 
promote the recovery of macular structure in vitreous hemorrhage patients ${ }^{[4,5]}$. However, it remains unclear what is the optimal timing of vitrectomy. This study is aimed to analyze the associations between the post-operation outcomes and variant timing of surgical intervention in the PDR patients with vitreous hemorrhage.

\section{Methods}

Clinical data This study included 76 patients (85 eyes) who were diagnosed with diabetic vitreous hemorrhage at the Eye Hospital of Wenzhou Medical University from June 2011 to October 2016. The inclusion criteria were as listed,

1) The patients who were diagnosed with diabetes with vitreous hemorrhage caused by diabetic retinopathy;

2) They received the vitrectomy for the vitreous hemorrhage;

3) The postoperative follow-up period was more than 3 months;

4) The ocular examinations were performed and recorded before and at one and three months after surgery, which included B-ultrasound, optical coherence tomography (OCT), fundus photography, best corrected visual acuity (BCVA) and slit lamp inspection.

The exclusion criteria are as following,

1) Severe cataract (worse than C2N2P2)

2) Iris neovascularization;

3) Secondary angiogenic glaucoma before surgery;

4) patients with diopter $\geq-6 \mathrm{D}$ or ocular axis $\geq 26.0 \mathrm{~mm}$.

Grouping The enrolled eyes were grouped based on the duration between the occurrence of vitreous hemorrhage and the time of vitrectomy. The date of the vitreous hemorrhage was determined according to the patient's chief complaint and the B-ultrasound scans. The four groups (Group 1 Group 4) had a time gap of less than 15 days (28 eyes from 27 patients), 15-30 days (29 eyes from 27 patients), 30-90 days (14 eyes from 11 patients), and more than 90 days (14 eyes from 11 patients) respectively.

Surgical methods All procedures were performed by an experienced surgeon under retrobulbar block after the blood glucose and the blood pressure were well managed. Standard three-channel $23 \mathrm{G}$ vitrectomy via the pars plana was performed to remove the opaque vitreous body, the posterior vitreous cortex, and the neovascular membrane. The fundus bleeding was treated with the intraocular electrocoagulation and photocoagulation. 
The filling materials were selected based on the fundus condition observed during the operation, which included the silicone oil, perfusate filling, inert gas (C3F8) or air filling. For details, silicone oil was mainly applied in the patients with traction retinal detachment and retinal stiffness who were not suitable for the complete panretinal photocoagulation, or in the patients with poor laser response, large retinal tears, or multiple retinal breaks; C3F8 or air was applied in patients with low risk of post-operation retinal detachment. Proper amount of the filling material was applied to keep the intraocular pressure normal.

Statistical methods The statistical analyses were performed using SPSS 22.0. Chi-square test was used to detect the differences in the gender ratio and the rate of preoperative laser treatment among different groups. The patients' phakic status, diabetes type, course of diabetes, preoperative intraocular anti-VEGF injection, intraoperative intima and proliferative membrane stripping, intraoperative intraocular filling, and postoperative complications between different groups were compared with Fisher's exact test. One-way ANOVA was used to compare the mean age, follow-up time, vitreous hemorrhage time, visual acuity and central macular thickness (CMT) of the four groups. The intra-group comparison was performed by paired t-test and other methods. The difference was statistically significant at $\mathrm{P}<0.05$.

\section{Results}

Patients' characteristics This study included 76 patients (85 eyes) who were diagnosed with diabetic vitreous hemorrhage, which consisted of 38 males (40 eyes) and 38 females (45 eyes). The patients' mean age was $55.8 \pm 13.9$ years (age range $25-79$ years) and the average follow-up time was $7.8 \pm 4.1$ months. Their mean course of diabetes was $12.3 \pm 7.4$ (range 1 30) years. Fifty patients were also diagnosed with hypertension. The BCVA was measured with the decimal visual chart, which was then converted to the scale of logarithm of minimal angle of resolution (logMAR). Light perception (LP), hand movement $(\mathrm{HM})$, and finger counting (FC) were recorded as 2.6, 2.3, and 1.85 logMAR respectively. (See Table 1)

Preoperative B-ultrasound scan classification and ophthalmic treatments The vitreous hemorrhage was classified into 3 types base on the pre-operation B-ultrasound scans, Type I: simple vitreous hemorrhage $\rrbracket$ Type II: vitreous hemorrhage with proliferative strips drawn from the ball wall $\otimes T y p e$ : vitreous hemorrhage with rhegmatogenous retinal detachment or traction retinal detachment. No significant difference in the B-ultrasound scan classification was found among the 4 groups.

Concerning the history of preoperative ophthalmic treatments, no significant difference between the four groups was found in the preoperative rates of intravitreal anti-VEGF injection and fundus laser therapy. (see Table 2)

Surgical details Before the vitrectomy, eighty-two out of the 85 included eyes were phakic eyes and three eyes were pseudophakic. Then forty-five eyes underwent cataract extraction during vitrectomy and all the other 36 eyes remained phakic during follow-up. There was no significant difference in the constituent ratio of patients who underwent combined operation among the four groups $(P=0.053)$. (see Table 3$)$ 
Visual acuity The preoperative BCVA of the four groups were $1.42 \pm 0.78,1.52 \pm 0.58,1.69 \pm 0.53$, and $1.89 \pm 0.50$, respectively. There was no significant difference between the 4 groups $(P=0.139)$. However, the mean BCVA of the four groups was significantly different at 1 month after surgery $(P=0.043)$, which was $0.74 \pm 0.43,0.77 \pm 0.38,1.19 \pm 0.64$, and $1.40 \pm 0.49$, respectively. We recorded the results of the pairwise comparisons between two groups in the following way, P12 stands for the P value between Group 1 and Group 2. Then we had $\mathrm{P} 12=0.634 \llbracket \mathrm{P} 13=0.016, \mathrm{P} 14 \rrbracket 0.001 \otimes \mathrm{P} 23=0.038 \otimes \mathrm{P} 24 \otimes 0.001 \rrbracket \mathrm{P} 34=0.262$. The mean BCVA of the four groups at 3 months after vitrectomy was $0.60 \pm 0.40,0.64 \pm 0.43,1.00 \pm 0.58$, and $1.43 \pm 0.54$, respectively. A significant improvement of the mean BCVA were found in every group compared to the corresponding vision data at 1 month after surgery. At 3 months after the vitrectomy, there was no significant difference between the 4 groups $(P=0.155)$. In the pairwise comparison analysis, $\mathrm{P} 12=0.724, \mathrm{P} 13=0.033, \mathrm{P} 14<0.001, \mathrm{P} 23=0.046, \mathrm{P} 24<0.001, \mathrm{P} 34=0.051 .$. (See Table 4)

Changes in the macular fovea thickness (CMT) (unit: $\mu \mathrm{m}$ ): The mean CMT of Group 1 Group 4 at 1month after surgery was $265.53 \pm 65.28,327.80 \pm 83.41,372.8 \pm 127.50$ and $313.07 \pm 61.72$, respectively. There were significant differences between the four groups $(P=0.001)$. Pairwise comparisons of the mean CMT between the four groups, $\mathrm{P}_{12}=0.005, \mathrm{P}_{13}<0.005, \mathrm{P}_{14}=0.075, \mathrm{P}_{23}=0.103, \mathrm{P}_{24}=0.591, \mathrm{P}_{34}=0.063$. The mean CMT of Group 1-Group 4 at 3 months after surgery was $228.57 \pm 43.64,286.76 \pm 84.90,313.57$ \pm 144.45 and $318.07 \pm 106.51$ respectively. There were significant differences between the four groups $(P=0.006)$. Pairwise comparison between the four groups at 3 months after surgery, $P 12=0.019 \square$ $\mathrm{P} 13=0.004, \mathrm{P} 14=0.003 \otimes \mathrm{P} 23=0.303 \otimes \mathrm{P} 24=0.266 \otimes \mathrm{P} 34=0.943$. The mean change in the macular fovea thickness of the four groups at 1 month and 3 months after operation was $-34.90 \pm 54.66,-41.02 \pm 53.52$, $-59.43 \pm 139.58$ and $5.14 \pm 72.20$, respectively. There was no significant difference in the mean CMT change between the four groups $(P=0.16)$. A pairwise comparison of the mean CMT change was then performed between the groups and the results were $P_{12}=0.765 \otimes P_{13}=0.466, P_{14}=0.117 ه P_{23}=0.466 \rrbracket$ $\mathrm{P}_{24}=0.070 \otimes \mathrm{P}_{34}=0.030$. (see Table 5)

Prevalence of complications and recurrence rate At one month after the operation, no severe complications such as ocular hemorrhage, infection and high intraocular pressure were observed in the four groups. During the 3 months follow-up after operation, no severe complications were spotted in Group 1 and Group 2. In Group 3, there were 3 cases of ocular hypertension and 1 case of neovascular glaucoma during the follow-up period. In group 4, there were 4 cases of ocular hypertension and 3 cases of neovascular glaucoma in the first three months after vitrectomy.

\section{Discussion}

Vitrectomy was firstly applied for treating proliferative diabetic retinopathy in the 1980 s. It can be used to remove opaque refractive stroma and release the traction of incomplete detached vitreous to the surface of retina ${ }^{[6]}$. However, it remains controversial to apply vitrectomy in the treatment of vitreous hemorrhage in the PDR patients. Early studies indicate that vitreous hemorrhage can be treated with conservative treatments to promote the absorption of hemorrhage. Therefore, vitreous surgery is usually not 
considered until the hemorrhage is not absorbed after 3 months ${ }^{[7]}$. However, emerged publications indicate earlier vitrectomy for vitreous hemorrhage in the PDR patients may bring benefits.

The main mechanism of vitrectomy in the treatment of vitreous hemorrhage is to remove intraocular hemorrhage, restore the normal anatomical structure of the retina by releasing the traction of vitreous to the retina, and reduce the risk of the occurrence of macular edema ${ }^{[8]}$. Rogem et al. ${ }^{[9]}$ points out that earlier surgery to remove intravitreal hemorrhage can protect photoreceptors from lipid peroxidation, restore or improve the prognostic visual acuity. Recent studies have shown that early vitrectomy can significantly improve the prognostic visual acuity and promote the recovery of retinal anatomy in patients with type 1 or type 2 diabetes mellitus ${ }^{[7,10]}$. Although early surgical treatment is recommended in the aforementioned studies, one month after the hemorrhage is commonly used as a limit for early vitrectomy. In this study, we compared the postoperative visual acuity, macular thickness and postoperation complications of the four groups from 15 days, 1 month and 3 months.

A large number of literatures have reported that early vitrectomy is beneficial to the improvement of visual acuity and visual function ${ }^{[11-13]}$. Janelle et al. ${ }^{[14]}$ showed that the visual acuity of patients with vitreous hemorrhage who received vitrectomy within 30 days was significantly better than that of patients with delayed surgery. Our study showed that the best corrected visual acuity of the four groups at 1 month and 3 months after the operation was significantly higher than that before the operation. However, after pairwise comparison between the four groups, the results showed that there was no significant difference between Group 1 and the Group 2, but there was significant difference between the other groups. Therefore, our study indicates that vitrectomy within 1 month can achieve better vision.

Early vitrectomy can promote the recovery of macular structure ${ }^{[8,15]}$. Sharma et al. ${ }^{[16]}$ showed that vitrectomy can effectively treat macular edema. In our study, the macular foveal thickness of the 4 groups decreased at 1 and 3 months after operation, and the differences between the 4 groups and within the groups were statistically significant $(P \otimes 0.005)$. Meanwhile, there was no significant difference between the four groups $(P>0.16)$. In this study, the mean CMT values at 1 month and 3 months after operation in the early glassy resection group (Group 1 and 2) were lower than those in the late operation group (Group 3 and 4), which indicates that the timing of the operation has an effect on the CMT value. There was no significant difference in CMT at 1 month and 3 months after operation, and there was no significant difference between the four groups, which indicates that the timing of the operation has little effect on the change of CMT after operation. To sum up, early vitrectomy can promote the recovery of the structure of the macular area of the retina.

Common complications after vitreous surgery include vitreous hemorrhage, postoperative ocular hypertension, neovascular glaucoma, iatrogenic holes, retinal detachment and retinal traction ${ }^{[6,17]}$. Early vitrectomy can reduce the prevalence of the complications; with the development of vitrectomy techniques and instruments, the success rate of vitrectomy has been greatly improved. Although $23 \mathrm{G}$ vitrectomy is not significantly different from $20 \mathrm{~g}$ and $25 \mathrm{G}$ in the success rate of operation and the recovery of visual acuity after operation, $23 \mathrm{~g}$ effectively reduces the replacement of intraoperative 
instruments, shortens the time of operation and improves the efficiency of operation, and reduces the incidence of postoperative complications ${ }^{[6,7,18,19]}$. In this study, $23 \mathrm{~g}$ vitrectomy was performed and no serious complications occurred during the first 1 month after the operation. Some studies have shown that retinal exposure to high concentration of oxygen can reduce retinal blood flow and has a direct and strong contractile effect on retinal blood vessels, thus inhibiting the proliferation of retinal neovascularization. Early vitrectomy for patients with vitreous hemorrhage can directly contact the retina with the oxidizing environment, which is conducive to the contraction of retinal blood vessels and reduces the chance of postoperative neovascular bleeding. Our study agrees with this. During the 3-month followup in this study, the prevalence of the complications in Groups 3 and 4 was significantly high compared to those in Groups 1 and 2.

Although most surgeons in this study prefer early treatment, other studies suggest that the surgical treatment of vitreous hemorrhage should be postponed to avoid active neovascularization and reduce the probability of postoperative complications, such as vitreous hemorrhage and neovascular glaucoma ${ }^{\text {[20] }}$. If vitreous hemorrhage is associated with retinal traction or detachment that does not involve the macula, conservative treatment like observing may be rational ${ }^{[2,21]}$. However, vitreous hemorrhage may be resolved through drug treatment, the absorbing process is related to the ischemic state of the retina, the circulation of blood vessels, the degree of vitreous hemorrhage and pre-operation retinal laser

photocoagulation ${ }^{[22]}$. When observing the absorption of blood accumulation, appearance of the traction retinal detachment or serious proliferation will directly affect the prognosis. In this study, the incidence of retinal detachment in the early vitrectomy group (Group 1 and 2) was significantly lower than that in the late operation group (Group 3 and 4). Therefore, our study advocates early vitrectomy concerning the post-operation complicaitions.

\section{Conclusion}

In conclusion, although the exact timing of surgery for vitreous hemorrhage in the PDR patients remains controversial, our study suggests early vitrectomy can achieve better postoperative visual acuity, promote the recovery of the macular structure and reduce the prevalence of the complications.

\section{Declaration}

\section{Ethical approval and consent to participate}

This study obtained the ethical approval from the Institutional Review Board of the Eye Hospital of Wenzhou Medical University. All the procedures performed were consistent with the tenets of the Declaration of Helsinki. Informed consent was obtained from all individual participants included in the study.

\section{Conflict of interest}


The authors declare that they have no conflict of interest.

\section{Availability of data and material}

All people in both groups voluntarily joined this study with informed consents. The data was "anonymized, " or stripped of identifying information.

\section{Consent for publication}

For all manuscripts that include details, images, or videos relating to an individual person, written informed consent for the publication of these details was obtained from their parent or legal guardian.

\section{Authors' contributions}

LL designed the study and was a major contributor in writing the manuscript; $Y C$ was a major contributor in writing the manuscript and analyzed and interpreted the data; LW analyzed and interpreted the patient data; LS made substantial contributions to the design of the work.

\section{Funding}

This study was funded by research grants from National key Research and Development Program of China (Grant No.2017YFC0111305). The funding organization had no role in the design or conduct of this research.

\section{Acknowledgements}

The authors thank Pingjun Chang for his helpful revisions to the manuscript.

\section{Abbreviations}

PDR-proliferative diabetic retinopathy; BCVA- Best corrected visual acuity; CMT- central macular thickness; OCT- optical coherence tomography; VEGF- vascular endothelial growth factor; logMAR-logarithm of minimal angle of resolution ; $\mathrm{LP}=$ Light perception; HM-hand movement; $\mathrm{FC}$-finger counting (FC)

\section{References}

1. Al-Halafi AM (2015) Outer retinal tubulation in diabetic macular edema following anti-VEGF treatment. Eye Vis (Lond) 2:9

2. Newman DK (2010) Surgical management of the late complications of proliferative diabetic retinopathy. Eye (Lond) 24:441-449

3. Hutton DW, Stein JD, Bressler NM, Jampol LM, Browning D, Glassman AR (2017) Cost-effectiveness of Intravitreous Ranibizumab Compared With Panretinal Photocoagulation for Proliferative Diabetic 
Retinopathy: Secondary Analysis From a Diabetic Retinopathy Clinical Research Network Randomized Clinical Trial. JAMA Ophthalmol 135:576-584

4. El AJ, Carvounis PE (2014) Current management of vitreous hemorrhage due to proliferative diabetic retinopathy. Int Ophthalmol Clin 54:141-153

5. Arevalo JF, Lasave AF, Kozak I, Al RS, Al KE, Maia M, Farah ME, Cutolo C, Brito M, Osorio C, Navarro P, Wu L, Berrocal MH, Morales-Canton V, Serrano MA, Graue-Wiechers F, Sabrosa NA, Alezzandrini AA, Gallego-Pinazo R (2019) Preoperative Bevacizumab for Tractional Retinal Detachment in Proliferative Diabetic Retinopathy: A Prospective Randomized Clinical Trial. Am J Ophthalmol 207:279-287

6. Berrocal MH, Acaba LA, Acaba A (2016) Surgery for Diabetic Eye Complications. Curr Diab Rep 16:99

7. Huang CH, Hsieh YT, Yang CM (2017) Vitrectomy for complications of proliferative diabetic retinopathy in young adults: clinical features and surgical outcomes. Graefes Arch Clin Exp Ophthalmol 255:863-871

8. Jackson TL, Nicod E, Angelis A, Grimaccia F, Pringle E, Kanavos P (2017) Pars plana vitrectomy for diabetic macular edema: A Systematic Review, Meta-Analysis, and Synthesis of Safety Literature. Retina 37:886-895

9. Rogers BS, Symons RC, Komeima K, Shen J, Xiao W, Swaim ME, Gong YY, Kachi S, Campochiaro PA (2007) Differential sensitivity of cones to iron-mediated oxidative damage. Invest Ophthalmol Vis Sci 48:438-445

10. Stefánsson E, Bek T, Porta M, Larsen N, Kristinsson JK, Agardh E (2000) Screening and prevention of diabetic blindness. Acta Ophthalmol Scand 78:374-385

11. Gupta V, Arevalo JF (2013) Surgical management of diabetic retinopathy. Middle East Afr J Ophthalmol 20:283-292

12. Schoenberger SD, Miller DM, Riemann CD, Foster RE, Sisk RA, Hutchins RK, Petersen MR (2011) Outcomes of 25-gauge pars plana vitrectomy in the surgical management of proliferative diabetic retinopathy. Ophthalmic Surg Lasers Imaging 42:474-480

13. Gupta B, Sivaprasad S, Wong R, Laidlaw A, Jackson TL, McHugh D, Williamson TH (2012) Visual and anatomical outcomes following vitrectomy for complications of diabetic retinopathy: the DRIVE UK study. Eye (Lond) 26:510-516

14. Fassbender JM, Ozkok A, Canter H, Schaal S (2016) A Comparison of Immediate and Delayed Vitrectomy for the Management of Vitreous Hemorrhage due to Proliferative Diabetic Retinopathy. Ophthalmic Surg Lasers Imaging Retina 47:35-41

15. Joussen AM, Joeres S (2007) Benefits and limitations in vitreoretinal surgery for proliferative diabetic retinopathy and macular edema. Dev Ophthalmol 39:69-87

16. Sharma T, Fong A, Lai TY, Lee V, Das S, Lam D (2016) Surgical treatment for diabetic vitreoretinal diseases: a review. Clin Exp Ophthalmol 44:340-354

17. Yorston D, Wickham L, Benson S, Bunce C, Sheard R, Charteris D (2008) Predictive clinical features and outcomes of vitrectomy for proliferative diabetic retinopathy. Br J Ophthalmol 92:365-368 
18. Celik E, Sever O, Horozoglu F, Yanyalı A (2016) Segmentation and removal of fibrovascular membranes with high-speed $23 \mathrm{G}$ transconjunctival sutureless vitrectomy, in severe proliferative diabetic retinopathy. Clin Ophthalmol 10:903-910

19. Park DH, Shin JP, Kim SY (2010) Comparison of clinical outcomes between 23-gauge and 20-gauge vitrectomy in patients with proliferative diabetic retinopathy. Retina 30:1662-1670

20. Higuchi A, Yamada H, Kawai E, Fujiseki Y, Wada M, Kiuchi K, Uchida Y, Minamino K, Matsumura M (2005) Vitrectomy for proliferative diabetic retinopathy. Nippon Ganka Gakkai Zasshi 109:134-141

21. Cruz-lñigo YJ, Acabá LA, Berrocal MH (2014) Surgical management of retinal diseases: proliferative diabetic retinopathy and traction retinal detachment. Dev Ophthalmol 54:196-203

22. El-Asrar AM, Al-Mezain HS (2011) Pharmacologic vitreolysis in diabetic retinopathy. Curr Pharm Biotechnol 12:406-409

\section{Tables}

Due to technical limitations, Tables 1 - 5 are only available for download from the Supplementary Files section.

\section{Supplementary Files}

This is a list of supplementary files associated with this preprint. Click to download.

- table.docx 